\title{
Ground state pseudoscalar mesons on the light front: From the light to heavy sector
}

\author{
Chao Shi $\odot,{ }^{1, *}$ Ming Li, ${ }^{1}$ Xurong Chen $\odot,{ }^{2,3}$ and Wenbao Jia ${ }^{1}$ \\ ${ }^{1}$ Department of Nuclear Science and Technology, Nanjing University of Aeronautics and Astronautics, \\ Nanjing 210016, China \\ ${ }^{2}$ Institute of Modern Physics, Chinese Academy of Sciences, Lanzhou 730000, China \\ ${ }^{3}$ Guangdong Provincial Key Laboratory of Nuclear Science, Institute of Quantum Matter, \\ South China Normal University, Guangzhou 510006, China
}

(Received 26 August 2021; accepted 11 October 2021; published 10 November 2021)

\begin{abstract}
We extract the leading Fock state light-front wave functions (LF-LFWFs) of both the light and heavy pseudoscalar mesons, e.g., the pion (at masses of 130,310 , and $690 \mathrm{MeV}$ ), $\eta_{c}$ and $\eta_{b}$, from their covariant Bethe-Salpeter wave functions within the rainbow-ladder truncation. It is shown that the LF-LFWFs get narrower in $x$ (the longitudinal momentum fraction of the meson carried by the quark) with the increasing current quark mass, and the leading twist parton distribution amplitudes inherit this feature. In the case of the pion, the LF-LFWFs only contribute about $30 \%$ of the total Fock state normalization, indicating the presence of significant higher Fock states within. In contrast, in the cases of the $\eta_{c}$ and $\eta_{b}$, the LF-LFWFs contribute more than $90 \%$, suggesting the $Q \bar{Q}$ valence Fock state truncation as a good approximation for heavy mesons. We thus study the three-dimensional parton distributions of the $\eta_{c}$ and $\eta_{b}$ with the unpolarized generalized parton distribution function (GPD) and the transverse momentum-dependent parton distribution function. Through the gravitational form factors in connection with the GPD, the mass radii of the $\eta_{c}$ and $\eta_{b}$ in the lightcone frame are determined to be $r_{E, \mathrm{LC}}^{\eta_{c}}=0.150$ and $r_{E, \mathrm{LC}}^{\eta_{b}}=0.089 \mathrm{fm}$, respectively.
\end{abstract}

DOI: 10.1103/PhysRevD.104.094016

\section{INTRODUCTION}

As a result of the Higgs mechanism [1,2], very large current quark mass differences abound in quantum chromodynamics $(\mathrm{QCD})$, resulting in diverse hadronic phenomenon in the light and heavy sectors. In hard hadronic processes, the quarks' partonic nature, which is directly associated with their current masses, is exposed. The partonic structure of the hadrons is thus of great interest, as it reveals the substructure of hadrons and is experimentally accessible through various hard exclusive and/or inclusive processes.

Theoretically, the hadrons' partonic structure is described in terms of various parton distributions. The parton distribution amplitude (PDA), for instance, is an important quantity that incorporates the internal nonperturbative dynamics within a QCD bound state. It serves as a soft input for the factorization of various hard exclusive processes, such as deeply virtual meson production $[3,4]$, B meson decay [5-7], and exclusive charmonium $J / \psi+\eta_{c}$

\footnotetext{
*cshi@nuaa.edu.cn
}

Published by the American Physical Society under the terms of the Creative Commons Attribution 4.0 International license. Further distribution of this work must maintain attribution to the author(s) and the published article's title, journal citation, and DOI. Funded by SCOAP . pair production in $e^{+} e^{-}$annihilation [8,9]. The determination of the PDAs rely heavily on nonperturbative QCD methods. In the light quark sector, phenomenological models and methods, i.e., QCD sum rule [10,11], lightfront holographic QCD [12], and the Dyson-SchwingerBethe-Salpeter equations method (DS-BSEs) [13-15] yield predictions. Meanwhile, lattice QCD has predicted its first one or two nontrivial moments [16-18]. Recently, with the help of large momentum effective theory (LaMET) [19], lattice QCD is giving much more information by charting the pointwise behavior of the PDAs [20,21]. Notably, agreement between lattice QCD and DSBSEs is found for the pion chiral-extrapolated to physical mass [22]. On the other hand, there is no lattice QCD result on PDAs in the heavy sector yet. Recently, it has been proposed that the B meson PDA can be determined from heavy quark effective theory, by combining the LaMET and the Euclidean lattice simulation technique [23]. As compared to light mesons, heavy mesons are arguably simpler as the quarks move much more slowly inside, so light-front potential models $[9,24]$ and nonrelativistic QCD are applicable $[25,26]$. Meanwhile, the QCD sum rule and Dyson-Schwinger equation (DSEs) also extend from the light sector to the heavy sector and give their predictions on heavy meson distribution amplitude (DAs) [7,27-30]. 
On the other hand, the parton distribution functions (PDFs) of hadrons and, in particular, their threedimensional extensions, the generalized parton distribution functions (GPDs) [3,31,32], and transverse momentumdependent parton distributions functions (TMDs) [33], have drawn much attention in recent years. The GPDs provide a unified description of the parton distribution in longitudinal momentum and transverse spatial coordinates $[34,35]$, while the TMDs incorporate the transverse motion of partons and their spin-orbit correlations [36,37]. Meanwhile, the GPDs are connected with hadron matrix elements of the energy-momentum tensor through $x$-weighted moments, which provide valuable information about the spin, energy, and pressure distributions within hadrons [32,38-41]. The GPDs and TMDs thus provide much more abundant information concerning the hadron's parton structure. Although the present focus on hadrons lies mostly in the light sector, e.g., the nucleon and pion/kaon mesons that are relatively stable [42-44], it is of theoretical interest to look into the 3D structure of the heavy hadrons with the help of GPDs and TMDs.

As light-cone quantities, the PDA, GPD, and TMD are interconnected by the light-front wave functions (or lightcone wave functions). The PDA is the leading Fock state light-front wave function (LF-LFWF) integrated over transverse momentum $\boldsymbol{k}_{T}$ [45], and the GPD and TMD can be calculated with overlap representations in terms of LFWFs [46-49]. The standard way to obtain the LFWFs is by diagonalizing the light-cone Hamiltonian. Challenges lie in the construction of the light-cone Hamiltonian in connection with QCD, as well as its diagonalization when more Fock states are involved [50]. Recently, with the help of the basis light-front quantization (BLFQ) technique, the $|q \bar{q} g\rangle$ state has been included in the pion and the calculation thus goes beyond the leading Fock state truncation [51]. On the other hand, an alternative approach exists by extracting the LFWFs from hadrons' covariant wave functions in the ordinary space-time frame, namely, the instant form [48,52-55]. Using this approach, we obtained the LFLFWFs of the pion and kaon [56,57], and later the vector mesons $\rho$ and $J / \psi$ [58]. A unique advantage of this approach is that it circumvents the light-cone Hamiltonian construction and diagonalization and allows the extraction of LFLFWFs from many Fock states. In this work, based on the study of the pion at the physical mass in [57], we will predict LF-LFWFs of fictitious pions at masses of 310 and $690 \mathrm{MeV}$ that are directly accessible by lattice QCD, as well as $\eta_{c}$ and $\eta_{b}$ from the heavy sector.

The light pseudoscalar meson sits in a special position as it is the Goldstone boson of dynamical chiral symmetry breaking (DCSB). The pion is thus dominated by the DCSB phenomenon, while the Higgs mechanism is almost irrelevant [59]. However, in the heavy mesons, the situation is the opposite: the Higgs mechanism generates most of the quark masses (and, consequently, the hadron mass), but the
DCSB effect weakens. The pseudoscalar mesons across the light and heavy sectors thus provide a good window to observe how the LF-LFWFs evolve with the strength shift between the DCSB and Higgs mechanism. The present study is therefore motivated in several directions: In the light quark sector, we study the LF-LFWFs of the pion at the physical mass $(\approx 130 \mathrm{MeV})$ and make predictions at testing masses of $m_{\pi}=310$ and $690 \mathrm{MeV}$. The latter two cases are chosen as they are directly accessible in lattice simulations [22]. Note that lately the authors of [60] have proposed a way to extract the hadron LFWFs through lattice QCD based on LaMET, so lattice results can be expected. In the heavy sector, we report the $\eta_{c}$ and $\eta_{b}$ LFLFWFs determined for the first time from the DS-BSEs. Using these LFWFs, we analyze the PDAs of $\eta_{c}$ and $\eta_{b}$ and investigate their 3D parton structure with the help of GPDs and TMDs.

This paper is organized as follows: In Sec. II, we introduce the DS-BSEs formalism and extract the LFLFWFs from the covariant BS wave functions. In Sec. III, we show the calculated LF-LFWFs of the pion (at different masses), $\eta_{c}$ and $\eta_{b}$, as well as their PDAs. A comparison is made between the light and heavy mesons. In Sec. IV, the parton structures of $\eta_{c}$ and $\eta_{b}$ are studied by means of the GPD (at zero skewness) and the unpolarized TMD. Finally, we conclude in Sec. V.

\section{FROM BETHE-SALPETER WAVE FUNCTIONS TO LF-LFWFs}

Within the DS-BSEs framework, the mesons are treated as bound states and described by their covariant BetheSalpeter (BS) wave functions. Within the rainbow-ladder (RL) truncation, which is taken throughout this work, the pseudoscalar mesons can be solved by aligning the quark's DSE for full quark propagator $S(k)$ and meson's BSE for BS amplitude $\Gamma_{M}(k, P)[61,62]$, i.e.,

$$
\begin{aligned}
S(k)^{-1}= & Z_{2}\left(i \gamma \cdot k+Z_{4} m(\mu)\right) \\
+ & Z_{2}^{2} \int_{\ell}^{\Lambda} \mathcal{G}(\ell) \ell^{2} D_{\mu \nu}^{\mathrm{free}}(\ell) \frac{\lambda^{a}}{2} \gamma_{\mu} S(k-\ell) \frac{\lambda^{a}}{2} \gamma_{\nu}, \quad(1) \\
\Gamma_{M}(k ; P)= & -Z_{2}^{2} \int_{q}^{\Lambda} \mathcal{G}\left((k-q)^{2}\right)(k-q)^{2} \\
& \times D_{\mu \nu}^{\mathrm{free}}(k-q) \frac{\lambda^{a}}{2} \gamma_{\mu} S\left(q_{+}\right) \Gamma_{M}(q ; P) S\left(q_{-}\right) \frac{\lambda^{a}}{2} \gamma_{\nu} .
\end{aligned}
$$

Here $\int_{q}^{\Lambda}$ implements a Poincaré invariant regularization of the four-dimensional integral, with $\Lambda$ the regularization mass scale. The $D_{\mu \nu}^{\text {free }}$ is the free gluon propagator in the Landau gauge. The quark momentum partition $q_{ \pm}=q \pm P / 2$. The $m(\mu)$ is the current quark mass renormalized at a scale of $\mu$. The $Z_{2}$ and $Z_{4}$ are the quark 
wave function and mass renormalization constants, respectively. Here a factor of $Z_{2}^{2}$ is picked out to preserve multiplicative renormalizability in solutions of the DSE and BSE [62]. The Bethe-Salpeter amplitudes are eventually normalized canonically

$$
\begin{aligned}
2 P_{\mu}= & \int_{k}^{\Lambda}\left\{\operatorname{Tr}\left[\bar{\Gamma}_{M}(k ;-P) \frac{\partial S\left(k_{+}\right)}{\partial P_{\mu}} \Gamma_{M}(k ; P) S\left(k_{-}\right)\right]\right. \\
& \left.+\operatorname{Tr}\left[\bar{\Gamma}_{M}(k ;-P) S\left(k_{+}\right) \Gamma_{M}(k ; P) \frac{\partial S\left(k_{-}\right)}{\partial P_{\mu}}\right]\right\},
\end{aligned}
$$

with $\quad \bar{\Gamma}_{M}(k,-P)^{T}=C^{-1} \Gamma_{M}(-k,-P) C$ and $C=\gamma_{2} \gamma_{4}$. Notably, the RL truncation preserves the (near) chiral symmetry of QCD by respecting the axial vector WardTakahashi identity [63]. The DCSB is therefore faithfully reflected and the Goldstone nature of light pseudoscalar mesons is manifest. Meanwhile, the RL truncation also applies to the heavy mesons [64-66]. We therefore solve for the pion and heavier $\eta_{c}$ and $\eta_{b}$ mesons in the same RL truncation.

The modeling function $\mathcal{G}\left(l^{2}\right)$ in Eqs. (1) and (2) absorbs the strong coupling constant $\alpha_{s}$, as well as the dressing effect in both the quark-gluon vertex and full gluon propagator. Popular models include the Maris-Tandy (MT) model and the later Qin-Chang (QC) model [67]

$\mathcal{G}(s)=\frac{8 \pi^{2}}{\omega^{4}} D \mathrm{e}^{-s / \omega^{2}}+\frac{8 \pi^{2} \gamma_{m}}{\ln \left[\tau+\left(1+s / \Lambda_{\mathrm{QCD}}^{2}\right)^{2}\right]} \mathcal{F}(s)$.

The second term in Eq. (4) is the perturbative QCD result $[61,67]$ that describes the UV behavior, while the first term incorporates essentially nonperturbative dressing effects at low and moderate momentum. As compared to the MT model, the QC model improves the far infrared behavior of the gluon propagator to be in line with lattice QCD [68,69] and modern DSE study [70], while for studying hadrons the two are equally good. Historically, combined with the RL truncation, the MT and/or QC models well describe a range of hadron properties, including the pseudoscalar and vector meson masses, decay constants, and various elastic and transition form factors [63,71-75].

The model parameters in this work are as follows. For the pion, we consider three cases, i.e., $m_{\pi}=130, m_{\pi}=310$, and $m_{\pi}=690 \mathrm{MeV}$. The first one is physical, while the latter two are for exploratory purposes, but directly accessible in lattice QCD simulations. In Eq. (4), we employ the welldetermined parameters $\omega=0.5 \mathrm{GeV}, D=(0.82 \mathrm{GeV})^{3} / \omega$ [67]. Meanwhile, we omit the UV term of Eq. (4), which determines the UV behavior of the pion's BS amplitude. Physically, this means we will only focus on the low and moderate $\boldsymbol{k}_{T}$ part of the pion's LF-LFWFs, but discard their UV part. In this case, Eqs. (1) and (2) are superrenormalizable and the renormalization constants $Z_{2}$ and $Z_{4}$ can be set to 1 . The only remaining parameter is the current quark mass and we take $m_{u / d}=5,27$, and $119 \mathrm{MeV}$, which produce $m_{\pi}=130,310$, and $690 \mathrm{MeV}$, respectively. Note that $m_{u / d}=119 \mathrm{MeV}$ already reaches the strange quark mass. In the heavy sector, we take $\omega=0.7 \mathrm{GeV}, D=$ $(0.765 \mathrm{GeV})^{3} / \omega$. They reproduce the physical mass spectrum and decay constants of heavy mesons as $\eta_{c}, J / \psi, \eta_{b}$, and $\Upsilon$. Their deviation from those in the light sector is a consequence of the diminishing of the dressing effect from the light antiquark-quark-gluon vertex $\bar{q} q g$ to the heavy $\bar{Q} Q g$ vertex [28]. For the mass parameters, we determine $M_{c}\left(m_{c}^{2}\right)=m_{c}=1.32$ and $M_{b}\left(m_{b}^{2}\right)=m_{b}=4.30 \mathrm{GeV}$. Here the $M_{c / b}\left(k^{2}\right)$ are the mass functions of the quark propagator defined in Eq. (5). These parameters produce $m_{\eta_{c}}=2.92$ and $m_{\eta_{b}}=9.4 \mathrm{GeV}$ and decay constants $f_{\eta_{c}}=$ 0.272 and $f_{\eta_{b}}=0.476 \mathrm{GeV}$. For comparison, we recall that the particle data group gives $m_{c}=1.27 \pm 0.02, m_{b}=$ $4.18_{-0.02}^{+0.03}, m_{\eta_{c}}=2.984$, and $m_{\eta_{b}}=9.398 \mathrm{GeV}$ [76]. For the decay constants, lattice QCD gives $f_{\eta_{c}}=0.279$ [77] and $f_{\eta_{b}}=0.472 \mathrm{GeV}$ [78], respectively.

Using these parameters, the $S(k)$ and $\Gamma_{M}(k ; P)$ can be numerically solved with Eqs. (1) and (2). Note that $S(k)$ admits the general decomposition

$$
S(k)=\frac{1}{i A\left(k^{2}\right) \not k+B\left(k^{2}\right)}=\frac{Z\left(k^{2}\right)}{i \not k+M\left(k^{2}\right)}
$$

and $\Gamma_{M}(k ; P)$ can be decomposed as

$$
\begin{aligned}
\Gamma_{M}(k ; P)= & \gamma_{5}[i E(k ; P)+\not P F(k ; P) \\
& +(k \cdot P) \not k G(k ; P)+i[\not k, P] H(k ; P)] .
\end{aligned}
$$

The $\mathcal{F}=E, F, G$, and $H$ are scalar functions of $k^{2}, k \cdot P$, and $P^{2}$. In the end, we obtain the numerical solutions for $A$, $B$, and $\mathcal{F}$ 's.

To extract the LF-LFWFs, we further parametrize $S(k)$ and $\Gamma_{M}(k ; P)$ with analytical forms. The $S(k)$ is written as the sum of pairs of complex conjugate poles [79]

$$
S(k)=\sum_{i=1}^{N}\left[\frac{z_{i}}{i \not k+m_{i}}+\frac{z_{i}^{*}}{i \not k+m_{i}^{*}}\right],
$$

with $N=2$. For the BS amplitude $\Gamma_{M}(k ; P)$, we parametrize its scalar functions $\mathcal{F}=E, F, G$, and $H$ with a Nakanishi-like representation $[57,80]$

$$
\begin{aligned}
\mathcal{F}(k ; P)= & \int_{-1}^{1} d \alpha \rho_{i}(\alpha)\left[\frac{U_{1} \Lambda^{2 n_{1}}}{\left(k^{2}+\alpha k \cdot P+\Lambda^{2}\right)^{n_{1}}}\right. \\
& \left.+\frac{U_{2} \Lambda^{2 n_{2}}}{\left(k^{2}+\alpha k \cdot P+\Lambda^{2}\right)^{n_{2}}}\right] \\
& +\int_{-1}^{1} d \alpha \rho_{u}(\alpha) \frac{U_{3} \Lambda^{2 n_{3}}}{\left(k^{2}+\alpha k \cdot P+\Lambda^{2}\right)^{n_{3}}},
\end{aligned}
$$




$$
\begin{aligned}
\rho_{i}(\alpha)= & \frac{1}{\sqrt{\pi}} \frac{\Gamma(3 / 2)}{\Gamma(1)}\left[C_{0}^{(1 / 2)}(\alpha)\right. \\
& \left.+\sigma_{1}^{i} C_{1}^{(1 / 2)}(\alpha)+\sigma_{2}^{i} C_{2}^{(1 / 2)}(\alpha)\right],
\end{aligned}
$$

where $\rho_{u}(\alpha)=\frac{3}{4}\left(1-\alpha^{2}\right)$ and $\left\{C_{n}^{(1 / 2)}, n=0,1, \ldots, \infty\right\}$ are the Gegenbauer polynomials of order $1 / 2$. The values of the parameters are listed in the Appendix. The outgoing quark and antiquark in the meson carry momentum $k+P / 2$ and $k-P / 2$, respectively, so $\mathcal{F}(k ; P)$ is even in $k \cdot P$ due to charge parity.

On the other hand, in the light-cone frame, the pseudoscalar meson $M$ with valence quark $f$ and valence antiquark $\bar{h}$ at the leading Fock state is given by $[50,81]$

$$
\begin{aligned}
|M\rangle= & \sum_{\lambda_{1}, \lambda_{2}} \int \frac{d^{2} \boldsymbol{k}_{T}}{(2 \pi)^{3}} \frac{d x}{2 \sqrt{x \bar{x}}} \frac{\delta_{i j}}{\sqrt{3}} \\
& \times \Phi_{\lambda_{1}, \lambda_{2}}\left(x, \boldsymbol{k}_{T}\right) b_{f, \lambda_{1}, i}^{\dagger}\left(x, \boldsymbol{k}_{T}\right) d_{h, \lambda_{2}, j}^{\dagger}\left(\bar{x}, \overline{\boldsymbol{k}}_{T}\right)|0\rangle,
\end{aligned}
$$

where $\boldsymbol{k}_{T}$ is the transverse momentum of the quark $f$, and $x=\frac{k^{+}}{P^{+}}$is the light-cone longitudinal momentum fraction of the active quark. The other variables are $\bar{x}=1-x$ and $\overline{\boldsymbol{k}}_{T}=-\boldsymbol{k}_{T}$. The $\lambda_{i}=(\uparrow, \downarrow)$ denotes the quark helicity and $\delta_{i j} / \sqrt{3}$ is the color factor. The $b^{\dagger}$ and $d^{\dagger}$ are the creation operators for a quark and antiquark, respectively. The $\Phi_{\lambda_{1}, \lambda_{2}}\left(x, \boldsymbol{k}_{T}\right)$ are the LFWFs that encode the nonperturbative internal dynamical information.

Meanwhile, in the case of pseudoscalar mesons, using the constraint from $\hat{Y}$ parity (the $\hat{Y}$ transform consists of a parity operation followed by a $180^{\circ}$ rotation around the $y$ axis [82]), the four $\Phi_{\lambda_{1}, \lambda_{2}}\left(x, \boldsymbol{k}_{T}\right)$ 's can be expressed with two independent scalar amplitudes [82], i.e.,

$$
\begin{array}{ll}
\Phi_{\uparrow, \downarrow}\left(x, \boldsymbol{k}_{T}\right)=\psi_{0}\left(x, \boldsymbol{k}_{T}^{2}\right), & \Phi_{\downarrow, \uparrow}\left(x, \boldsymbol{k}_{T}\right)=-\psi_{0}\left(x, \boldsymbol{k}_{T}^{2}\right), \\
\Phi_{\uparrow, \uparrow}\left(x, \boldsymbol{k}_{T}\right)=k_{T}^{-} \psi_{1}\left(x, \boldsymbol{k}_{T}^{2}\right), & \Phi_{\downarrow, \downarrow}\left(x, \boldsymbol{k}_{T}\right)=k_{T}^{+} \psi_{1}\left(x, \boldsymbol{k}_{T}^{2}\right),
\end{array}
$$

where $k_{T}^{ \pm}=k^{1} \pm i k^{2}$. The subscripts 0 and 1 of $\psi\left(x, \boldsymbol{k}_{T}^{2}\right)$ indicate the orbital angular momentum of the (anti)quark projected onto the light-cone $z$ direction. The $\psi\left(x, \boldsymbol{k}_{T}^{2}\right)$ 's are easier to compute as they contain fewer variables than $\Phi\left(x, \boldsymbol{k}_{T}\right)$. They can be obtained from the Bethe-Salpeter wave function via the light-front projections $[56,83,84]$

$$
\begin{aligned}
\psi_{0}\left(x, \boldsymbol{k}_{T}^{2}\right)= & \sqrt{3} i \int \frac{d k^{+} d k^{-}}{2 \pi} \\
& \times \operatorname{Tr}_{D}\left[\gamma^{+} \gamma_{5} \chi_{f \bar{h}}(k, P)\right] \delta\left(x P^{+}-k^{+}\right), \\
\psi_{1}\left(x, \boldsymbol{k}_{T}^{2}\right)= & -\sqrt{3} i \int \frac{d k^{+} d k^{-}}{2 \pi} \frac{1}{\boldsymbol{k}_{T}^{2}} \\
& \times \operatorname{Tr}_{D}\left[i \sigma^{+i} \boldsymbol{k}_{T i} \gamma_{5} \chi_{f \bar{h}}(k, P)\right] \delta\left(x P^{+}-k^{+}\right),
\end{aligned}
$$

where $\sigma^{+i}=\frac{i}{2}\left[\gamma^{+}, \gamma^{i}\right]$ and the plus component of a four vector $A$ is $A^{+}=\left(A^{0}+A^{3}\right) / \sqrt{2}$. The trace is taken over Dirac indices. The BS wave function can be expressed using the quark propagator $S(k)$ and the BS amplitude $\Gamma_{M}(k ; P)$ as $\chi_{f \bar{h}}(k ; P)=S_{f}(k+P / 2) \Gamma_{M}(k ; P) S_{h}(k-P / 2)$. Using Eqs. (7)-(9) and Eqs. (12) and (13), one can reproduce the pointwise behavior of $\psi\left(x, \boldsymbol{k}_{T}^{2}\right)$ with very high precision, using the method explained in Sec. II of [57].

\section{LF-LFWFs OF PSEUDOSCALAR MESONS}

The LF-LFWFs for the pion are shown in Fig. 1. From the top row to the bottom, we display the results of $m_{\pi}=130,310$, and $690 \mathrm{MeV}$, respectively. Comparing these LF-LFWFs, a prominent feature is that, as the current quark mass increases, the LF-LFWFs get narrower in $x$. Figure 1 therefore suggests that the DCSB tends to broaden the $x$ distribution of pseudoscalar mesons LF-LFWFs, while the explicit chiral symmetry breaking brought by the Higgs mechanism does the opposite. The tendency continues to the heavy sector. In Fig. 2, we show the LFLFWFs of $\eta_{c}$ and $\eta_{b}$ mesons, which are significantly narrower than those in the light quark sector. On the other hand, the light meson LF-LFWFs decrease much faster than the heavy meson in $\boldsymbol{k}_{T}$. This indicates the transverse motion of quarks in heavy mesons are more active than that in the light system.

Aside from the profile, the magnitude of the LF-LFWFs also provide important information of the pseudoscalar mesons' parton structure. Rigorously speaking, the meson's LFWFs of all Fock states should normalize to unity, i.e.,

$$
1=\sum_{\lambda, \lambda^{\prime}} N_{\lambda, \lambda^{\prime}}+N_{\mathrm{HF}}
$$
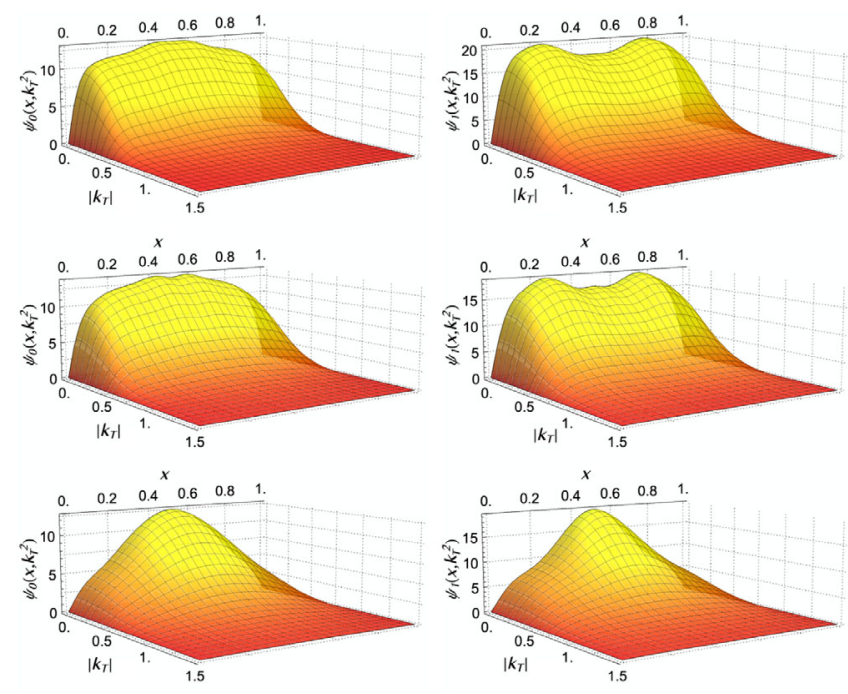

FIG. 1. The $\psi_{0}\left(x, \boldsymbol{k}_{T}^{2}\right)$ and $\psi_{1}\left(x, \boldsymbol{k}_{T}^{2}\right)$ of pion at $m_{\pi}=130$ (top row), 310 (middle row), and $690 \mathrm{MeV}$ (bottom row). 

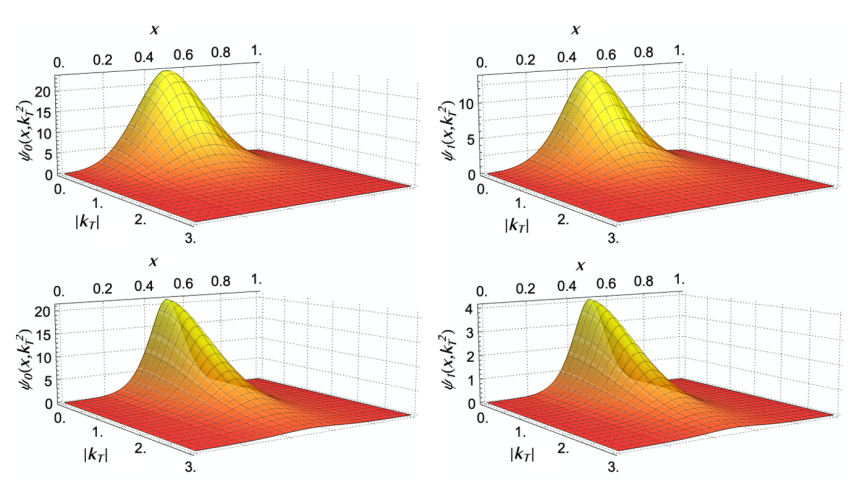

FIG. 2. The $\psi_{0}\left(x, \boldsymbol{k}_{T}^{2}\right)$ and $\psi_{1}\left(x, \boldsymbol{k}_{T}^{2}\right)$ of $\eta_{c}$ (top row) and $\eta_{b}$ (bottom row).

with

$$
N_{\lambda, \lambda^{\prime}}=\int_{0}^{1} d x \int \frac{d \boldsymbol{k}_{T}^{2}}{2(2 \pi)^{3}}\left|\Phi_{\lambda, \lambda^{\prime}}\left(x, \boldsymbol{k}_{T}\right)\right|^{2} .
$$

The $N_{\mathrm{HF}}$ refers to higher Fock states contribution. The $N$ 's are simply the overlap between the LFWFs and their complex conjugate, so they are positive definite. Here we infer the value of $N_{\mathrm{HF}}$ by subtracting unity with the LFLFWFs contribution. Our result is listed in Table I. Apparently, as the system gets heavier, the higher Fock states contribution diminishes. This suggests (i) heavy systems such as $c \bar{c}$ and $b \bar{b}$ could be well approximated with their LF-LFWFs and (ii) in a light system such as the pion where the Higgs mechanism is almost irrelevant, there are lots of higher Fock states generated in association with the DCSB. We therefore believe this is another novel property of the parton structure of mesons in connection with the DCSB.

Finally, there is a shift in the relative strength between $\psi_{0}\left(x, \boldsymbol{k}_{T}^{2}\right)$ and $\psi_{1}\left(x, \boldsymbol{k}_{T}^{2}\right)$ as the quark mass changes. Defining the ratio

$$
r \equiv \frac{N_{\uparrow, \uparrow}+N_{\downarrow, \downarrow}}{N_{\uparrow, \downarrow}+N_{\downarrow, \uparrow}}=\frac{N_{\uparrow, \uparrow}}{N_{\uparrow, \downarrow}},
$$

we find $r=0.56,0.16$, and 0.04 for pion, $\eta_{c}$, and $\eta_{b}$ respectively. Therefore as the spin antiparallel ( $\mathrm{S}$-wave) LFWF $\psi_{0}\left(x, \boldsymbol{k}_{T}^{2}\right)$ provides a larger contribution, there is also a considerable P-wave component in the pion. In

TABLE I. LFWFs contribution to Fock states normalization. See Eq. (15) for definition of $N$ and Eq. (16) for $r$.

\begin{tabular}{lcccc}
\hline \hline & $N_{\uparrow, \downarrow}=N_{\downarrow, \uparrow}$ & $N_{\uparrow, \uparrow}=N_{\downarrow, \downarrow}$ & $N_{\mathrm{HF}}$ & $r$ \\
\hline$\pi(130 \mathrm{MeV})$ & 0.1 & 0.056 & 0.69 & 0.56 \\
$\eta_{c}$ & 0.40 & 0.064 & 0.07 & 0.16 \\
$\eta_{b}$ & 0.48 & 0.02 & $\approx 0.0$ & 0.04 \\
\hline \hline
\end{tabular}

heavy quarkonium, the S-wave component becomes dominant as the system gets nonrelativistic.

We next look at the leading-twist parton distribution amplitudes of the mesons. The twist-2 PDA of pseudoscalar meson was originally defined as the $\boldsymbol{k}_{T}$-integrated LFWF [45], i.e.,

$$
\phi_{\pi}(x, Q) \propto \int_{\boldsymbol{k}_{T}^{2} \leq Q^{2}} \frac{d^{2} \boldsymbol{k}_{T}}{16 \pi^{3}} \psi_{0}\left(x, \boldsymbol{k}_{T}^{2}\right)
$$

with the normalization condition

$$
\int_{0}^{1} d x \phi(x, Q)=1
$$

In Fig. 3, we show our results for pion DAs for three masses, i.e., 130, 310, and $690 \mathrm{MeV}$, denoted by solid, dashed, and dotted curves, respectively. The green, red, and blue bands are adopted from lattice QCD calculation that employed the same pion masses, respectively, while at a hadron momentum of $P_{z}=1.75 \mathrm{GeV}$ in the LaMET approach [22]. General agreement between the two calculations is found, including the evolution of PDA with increasing current quark mass (or pion mass). For instance, the PDAs of the pion with mass 130 and $310 \mathrm{MeV}$ do not differ much, i.e., they are broad and concave functions. On the other hand, at a mass of $690 \mathrm{MeV}$, the PDAs get significantly narrower and are close to the asymptotic form $6 x(1-x)$ (gray dot-dash-dashed curve), as first pointed out in DS-BSEs [28] and confirmed later in [22]. We note that our PDAs are associated with a scale around $1.0 \mathrm{GeV}$ : since we only took the first term in Eq. (4), the model implements a soft momentum cutoff $\approx \Lambda \approx 2 \omega=1.0 \mathrm{GeV}$. Meanwhile the lattice QCD result is at the scale of $2 \mathrm{GeV}$. Under the Efremov-Radyushkin-Brodsky-Lepage (ERBL)

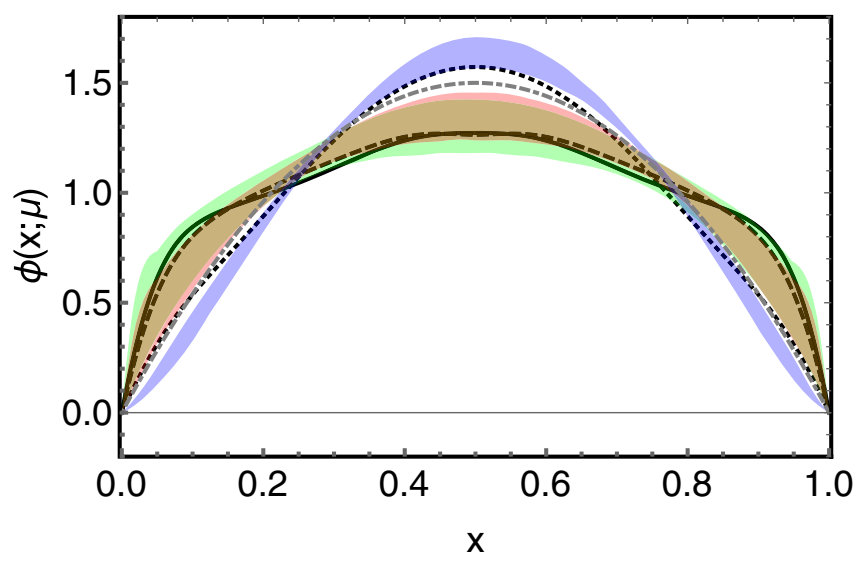

FIG. 3. The PDA of the pion at masses of $m_{\pi}=130$ (solid), 310 (dashed), and $690 \mathrm{MeV}$ (dotted). The colored bands are results from lattice QCD [22] at the same masses of $m_{\pi}=130$ (red), 310 (green), and $690 \mathrm{MeV}$ (blue). 


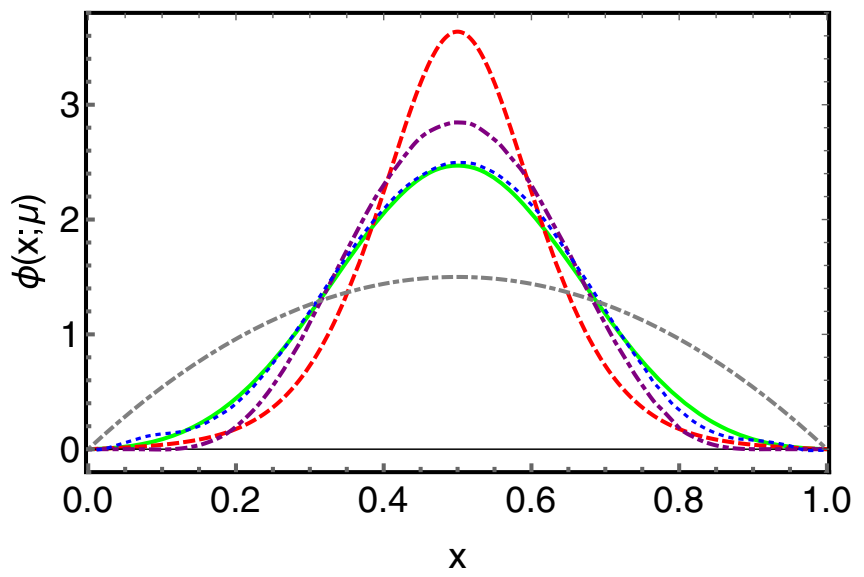

FIG. 4. A comparison of our PDA of $\eta_{c}$ (green solid) and $\eta_{b}$ (red dashed) and sum rule results (blue dotted and purple dotdashed, respectively) using background field theory [7].

evolution [45,85], all curves would evolve very slowly toward the asymptotic form.

The PDAs of $\eta_{c}$ and $\eta_{b}$ are shown in Fig. 4. The green solid curve is our result for $\phi_{\eta_{c}}\left(x, Q=m_{c}\right)$, with $m_{c}=1.32 \mathrm{GeV}$ in our parameter setup. The dashed red curve is our $\phi_{\eta_{b}}\left(x, Q=m_{b}\right)$ with $m_{b}=4.28 \mathrm{GeV}$. For comparison, we show the $\phi_{\eta_{c}}\left(x, Q \approx m_{c}\right)$ (blue dotted) and $\phi_{\eta_{b}}\left(x, Q \approx m_{b}\right)$ (purple dot-dashed) from the sum rule calculation of [7]. One can see the PDAs of $\eta_{c}$ agree very well. For $\eta_{b}$, there is some deviation between the two curves. However, the deviation reduces if the uncertainties are considered. For instance, in [7] the moments $\left\langle(2 x-1)^{m}\right\rangle$ with $m=2,4$, and 6 are $0.067 \pm 0.007$, $0.011 \pm 0.002$, and $0.003 \pm 0.001$, respectively, while our red dashed curve gives $0.059,0.0125$, and 0.0047 . On the other hand, the covariant light-front model [86] and BLFQ approach [24] all yield semiquantitatively similar results. In the absence of lattice QCD calculations, they all suggest that the PDAs of heavy pseudoscalar mesons are narrowly distributed in $x$ as compared to asymptotic form $6 x(1-x)$ (gray dot-dash-dashed curve).

\section{SPATIAL AND MOMENTUM TOMOGRAPHY OF THE HEAVY PSEUDOSCALAR MESONS}

In the light-cone gauge, the unpolarized quark GPD of a pseudoscalar meson is defined as

$$
\begin{aligned}
H_{M}^{q}(x, \xi, t ; \mu)= & \frac{1}{2} \int \frac{d z^{-}}{2 \pi} e^{i x P^{+} z^{-}} \\
& \times\left\langle P+\frac{\Delta}{2}\left|\bar{\psi}^{q}\left(-\frac{z^{-}}{2}\right) \gamma^{+} \psi^{q}\left(\frac{z^{-}}{2}\right)\right| P-\frac{\Delta}{2}\right\rangle .
\end{aligned}
$$

The $x$ is the parton's averaged light-cone momentum fraction and $\xi=-\frac{\Delta^{+}}{2 P^{+}}$is the skewness. The momentum transfer is $t=\Delta^{2}=-\frac{4 \xi^{2} m_{M}^{2}+\Delta_{T}^{2}}{1-\xi^{2}}$. The GPD has two distinct domains, where $|x|<|\xi|$ is the ERBL region and $1>|x|>|\xi|$ is the Dokshitzer-Gribov-Lipatov-Altarelli-Parisi region, named after their evolution with factorization scale $\mu$. Here we will focus on the GPD of $\eta_{c}$ and $\eta_{b}$ at zero skewness, i.e., the $H_{M}^{q}(x, 0, t)$. It gives rise to many interesting quantities, e.g., the one-dimensional collinear PDF, the impact parameter-dependent parton distributions (IPDs), and the gravitational form factor. The overlap representation of $H_{M}^{f}(x, 0, t)$ in terms of LFWFs reads $[46,47,83,87]$

$$
\begin{aligned}
H_{M}^{q}\left(x, 0, t ; \mu_{0}\right)= & \int \frac{d^{2} \boldsymbol{k}_{T}}{(2 \pi)^{3}}\left[\psi_{0}^{*}\left(x, \hat{\boldsymbol{k}}_{T}\right) \psi_{0}\left(x, \tilde{\boldsymbol{k}}_{T}\right)\right. \\
& \left.+\hat{\boldsymbol{k}}_{T} \cdot \tilde{\boldsymbol{k}}_{T} \psi_{1}^{*}\left(x, \hat{\boldsymbol{k}}_{T}\right) \psi_{1}\left(x, \tilde{\boldsymbol{k}}_{T}\right)\right],
\end{aligned}
$$

with $\hat{\boldsymbol{k}}_{T}=\boldsymbol{k}_{T}+(1-x) \frac{\boldsymbol{\Delta}_{T}}{2}$ and $\tilde{\boldsymbol{k}}_{T}=\boldsymbol{k}_{T}-(1-x) \frac{\boldsymbol{\Delta}_{T}}{2}$.

We plot $H_{\eta_{c}}^{c}\left(x, 0, t ; \mu_{0}\right)$ (yellow surface) and $H_{\eta_{b}}^{b}\left(x, 0, t ; \mu_{0}\right)$ (purple surface) in Fig. 5. Here we rescale the LF-LFWFs of $\eta_{c}$ and $\eta_{b}$ in Eqs. (12) and (13) by an overall factor so that the PDF, which relates to the GPD as $f_{M}^{q}\left(x ; \mu_{0}\right)=H_{M}^{q}\left(x, 0,0 ; \mu_{0}\right)$, is normalized to unity, i.e., $\int_{0}^{1} d x H_{M}^{q}\left(x, 0,0 ; \mu_{0}\right)=\int_{0}^{1} d x f_{M}^{q}\left(x ; \mu_{0}\right)=1$. This approximation is based on the finding that higher Fock states contribute little to the normalization, as shown in Table I. However, the value of $\mu_{0}$ is unknown. Phenomenologically, it is usually determined by comparing the first moment of the PDF with experiment or lattice QCD $[88,89]$, but this is infeasible in the heavy sector due to the lack of experiment data. In this paper, we follow [90] and presume that $\mu_{0}$ is around $2 m_{q}$. That corresponds to $\mu_{0} \approx 2.6 \mathrm{GeV}$ for $\eta_{c}$ and $\mu_{0} \approx 8.6 \mathrm{GeV}$ for $\eta_{b}$, close to the natural $\eta_{c}$ and $\eta_{b}$ mass scales. We recall that the various parton distributions studied below are all implicitly associated with such a scale, which will not be written explicitly.

The two-dimensional Fourier transform of $H_{M}^{q}\left(x, 0, \Delta_{T}^{2}\right)$ gives the IPD GPD

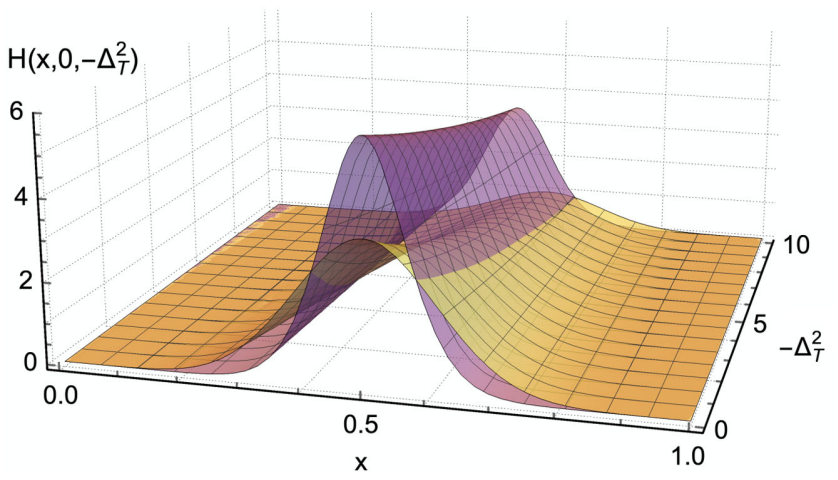

FIG. 5. The unpolarized GPD $H_{M}^{q}(x, \xi=0, t)$ of $\eta_{c}$ (yellow surface lower at $x=0.5$ ) and $\eta_{b}$ (purple surface upper at $x=0.5$ ) at scales around their masses. 

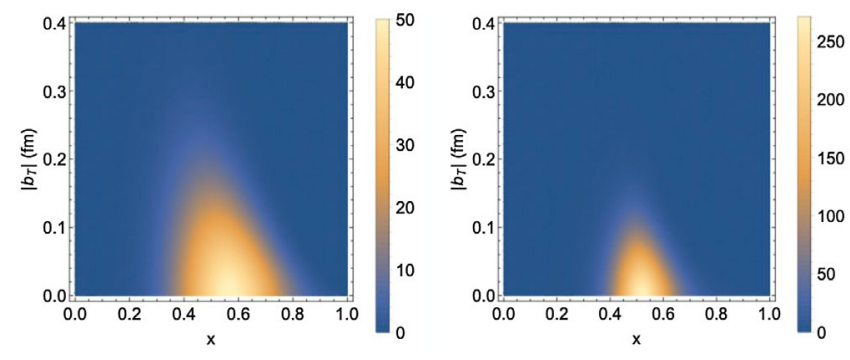

FIG. 6. The impact parameter-dependent GPD $\rho_{M}^{q}\left(x, \boldsymbol{b}_{T}^{2}\right)$ of $\eta_{c}$ and $\eta_{b}$. The $\rho_{\eta_{c}}^{c}\left(x, \boldsymbol{b}_{T}^{2}\right)$ is on the left column and $\rho_{\eta_{b}}^{b}\left(x, \boldsymbol{b}_{T}^{2}\right)$ on the right.

$$
\rho_{M}^{q}\left(x, \boldsymbol{b}_{T}^{2}\right)=\int \frac{d^{2} \boldsymbol{\Delta}_{T}}{(2 \pi)^{2}} H_{M}^{q}\left(x, 0,-\Delta_{T}^{2}\right) e^{i \mathbf{b}_{T} \cdot \Delta_{T}},
$$

which characterizes the probability density of partons on the transverse plane with the light-cone momentum fraction $x$ and the impact parameter $\boldsymbol{b}_{T}$ [35]. The $\boldsymbol{b}_{T}$ is defined as the separation between the active parton and the origin of transverse center of momentum, i.e., $\boldsymbol{b}_{T} \equiv \boldsymbol{b}_{T, 1}=\boldsymbol{r}_{T, 1}-\boldsymbol{R}_{T}$. In a two-body picture, $\boldsymbol{R}_{T}=x \boldsymbol{r}_{T, 1}+(1-x) \boldsymbol{r}_{T, 2}$, with $\boldsymbol{r}_{T, i}$ the transverse position of $i$ th parton. We show our results in Fig. 6, with the left column devoted to the charm quark in $\eta_{c}$ and the right column to the bottom quark in $\eta_{b}$. The charm quark in $\eta_{c}$ is more broadly distributed in both $x$ and impact parameter $\boldsymbol{b}_{T}$ as compared to the bottom quark in $\eta_{b}$ at their hadronic scales. We then integrate the $x$ dependence in the IPDs and obtain their spatial parton distributions $\rho_{M}^{q,(0)}\left(\boldsymbol{b}_{T}\right)=\int_{0}^{1} d x \rho_{M}^{q}\left(x, \boldsymbol{b}_{T}{ }^{2}\right)$. The result is plotted in the upper panel of Fig. 7 and the lower panel displays their
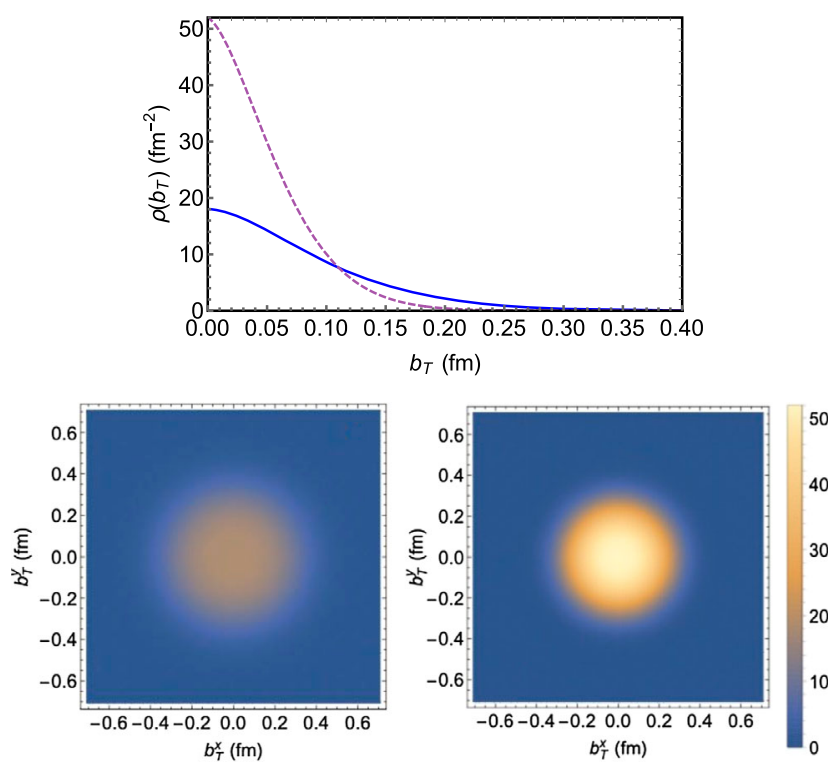

FIG. 7. Upper: the transverse spatial distribution of the charm quark within $\eta_{c}$ (blue solid) and the bottom quark within $\eta_{b}$ (purple dashed) on the light front. Lower: the density plots of the spatial distribution, with $\eta_{c}$ on the left and $\eta_{b}$ on the right. density plots. We determine their mean squared impact parameters $\left\langle\boldsymbol{b}_{T}^{2}\right\rangle^{q}=\int d \boldsymbol{b}_{T}^{2} \boldsymbol{b}_{T}^{2} \rho^{q,(0)}\left(\boldsymbol{b}_{T}^{2}\right)$ to be $\left\langle\boldsymbol{b}_{T}^{2}\right\rangle_{\eta_{c}}^{c}=$ $(0.157 \mathrm{fm})^{2}$ and $\left\langle\boldsymbol{b}_{T}^{2}\right\rangle_{\eta_{b}}^{b}=(0.092 \mathrm{fm})^{2}$. Comparing with our results on the pion and kaon $\left\langle\boldsymbol{b}_{T}^{2}\right\rangle_{\pi}^{u}=(0.332 \mathrm{fm})^{2}$, $\left\langle\boldsymbol{b}_{T}^{2}\right\rangle_{K}^{u}=(0.361 \mathrm{fm})^{2}$, and $\left\langle\boldsymbol{b}_{T}^{2}\right\rangle_{K}^{s}=(0.283 \mathrm{fm})^{2}$ from [57], one finds the heavy mesons are considerably more compact in the transverse plane on the light front.

By definition, the $\left\langle\boldsymbol{b}_{T}^{2}\right\rangle_{M}^{q}$ can be regarded as the square of quark distribution radius in the light-cone frame. Within the leading Fock state truncation and for a charged pseudoscalar meson, for instance, the $\pi^{+}$, the light-cone charge radius of $\pi^{+}$is $\left\langle r_{c, L C}^{2}\right\rangle_{\pi^{+}}=e_{u}\left\langle\boldsymbol{b}_{T}^{2}\right\rangle_{\pi^{+}}^{u}+e_{\bar{d}}\left\langle\boldsymbol{b}_{T}^{2}\right\rangle_{\pi^{+}}^{\bar{d}}=\left\langle\boldsymbol{b}_{T}^{2}\right\rangle_{\pi^{+}}^{u}$. It is related to the $\pi^{+}$charge radius in the Breit frame as $\left\langle r_{c, B r}^{2}\right\rangle_{\pi^{+}}=\frac{3}{2}\left\langle r_{c, L C}^{2}\right\rangle_{\pi^{+}}$. In heavy quarkonium, the charge radius vanishes for charge conjugation. So we can follow [24,91] and define a fictitious charge radius for $\eta_{c}$ in the Breit frame as $\left\langle r_{c, B r}^{2}\right\rangle_{\eta_{c}}=\frac{3}{2}\left\langle\boldsymbol{b}_{T}^{2}\right\rangle_{\eta_{c}}^{c}=(0.192 \mathrm{fm})^{2}$. Note that the standard DS-BSEs computation yields $\left\langle r_{c, B r}^{2}\right\rangle_{\eta_{c}}=$ $(0.219 \mathrm{fm})^{2}$ [91], in which infinitely many dressing and loop Feynman diagrams are calculated, thus higher Fock states are incorporated. In this sense, the deviation between 0.192 and $0.219 \mathrm{fm}$ can be due to the higher Fock states. Nevertheless, the deviation is less than $15 \%$, suggesting roughly the error brought by the leading Fock state truncation in $\eta_{c}$. We anticipate the deviation would be even smaller for heavier $\eta_{b}$.

The gravitational form factor of $\eta_{c}$ and $\eta_{b}$ can be connected with the $x$ moment of the GPD at $\xi=0$, i.e.,

$$
\int_{-1}^{1} d x x H^{q}(x, 0, t)=A_{2,0}^{q}(t) .
$$

The $A_{2,0}^{q}(t)$ denotes the quark's contribution to the hadron's gravitational form factor $A(t)$, which enters the general decomposition of the matrix element of the energy-momentum tensor (EMT) of spin-0 states [92-94]

$$
\begin{aligned}
\left\langle M\left(p^{\prime}\right)\left|T^{\mu \nu}(0)\right| M(p)\right\rangle= & \frac{1}{2}\left[P^{\mu} P^{\nu} A(t)\right. \\
& \left.+\left(g^{\mu \nu} q^{2}-q^{\mu} q^{\nu}\right) C(t)\right],
\end{aligned}
$$

with $P=p+p^{\prime}, q=p^{\prime}-p$, and $t=q^{2}$. The $A(t)$ is related to the $x$ moment of GPDs by $A(t)=\sum_{a} A_{2,0}^{a}(t ; \mu)$, where the $a=q, g$ runs through all partons. While the individual parton contributions $A_{2,0}^{a}(t ; \mu)$ are scale dependent, their summation $A(t)$ is not. In our case, as we have approximated the $\eta_{c}$ and $\eta_{b}$ solely with the $q \bar{q}$ Fock state, the gluon contribution $A_{2,0}^{g}\left(t ; \mu_{0}\right)$ vanishes at the initial scale $\mu_{0}$, thus $A(t)=2 A_{2,0}^{q}\left(t ; \mu_{0}\right)$. We show our results for $\eta_{c}$ and $\eta_{b}$ in Fig. 8.

The light-cone energy radius (or mass radius) $\left\langle r_{E, \mathrm{LC}}^{2}\right\rangle$ can be defined as the mean value of $\boldsymbol{r}_{\perp}^{2}$ weighted by the EMT in the light-cone frame, namely, $\frac{1}{2 P^{+}}\left\langle T^{++}\right\rangle_{\mathrm{LC}}\left(\boldsymbol{r}_{\perp}\right)$, where 


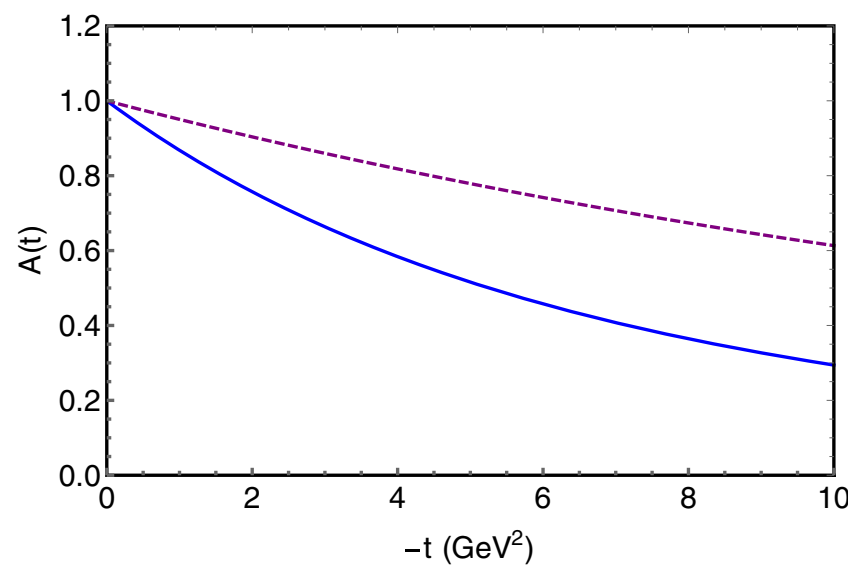

FIG. 8. The gravitational form factor $A(t)$ [defined in Eq. (23)] of $\eta_{c}$ (blue solid) and $\eta_{b}$ (purple dotted) in the leading Fock state truncation.

$$
\left\langle T_{\mu \nu}\right\rangle_{\mathrm{LC}}\left(\boldsymbol{r}_{\perp}\right)=\int \frac{d^{2} \Delta_{\perp}}{2 P^{+}(2 \pi)^{2}} e^{-i \Delta_{\perp} \boldsymbol{r}_{\perp}}\left\langle p^{\prime}\left|T_{\mu \nu}(0)\right| p\right\rangle .
$$

It is thus related to the gravitational form factor $A(t)$ by [95]

$$
\left\langle r_{E, \mathrm{LC}}^{2}\right\rangle=-\left.4 \frac{\partial A\left(Q^{2}\right)}{\partial Q^{2}}\right|_{Q^{2}=0},
$$

with $Q^{2}=-t$. For $\eta_{c}$ and $\eta_{b}$, we find the values to be $\left.\left\langle r_{E, \mathrm{LC}}^{2}\right\rangle_{\eta_{c}}=(0.150 \mathrm{fm})^{2}\right)$ and $\left\langle r_{E, \mathrm{LC}}^{2}\right\rangle_{\eta_{b}}=(0.089 \mathrm{fm})^{2}$, respectively. It is interesting to compare them with the quark distribution radius $\left\langle\boldsymbol{b}_{T}^{2}\right\rangle_{\eta_{c}}^{c}=(0.157 \mathrm{fm})^{2}$ and $\left\langle\boldsymbol{b}_{T}^{2}\right\rangle_{\eta_{b}}^{b}=(0.092 \mathrm{fm})^{2}$ above. The two radii are very close, with the light-cone energy radius a bit smaller. Such behavior is in agreement with the finding of [24] in the case of heavy mesons.

We finally investigate the transverse momentum distribution of quarks within $\eta_{c}$ and $\eta_{b}$. The unpolarized leadingtwist TMD of pseudoscalar meson is defined as

$$
\begin{aligned}
f_{1}\left(x, \boldsymbol{k}_{T}^{2}\right)= & \int \frac{d \xi^{-} d^{2} \boldsymbol{\xi}_{T}}{(2 \pi)^{3}} e^{i\left(\xi^{-} k^{+}-\boldsymbol{\xi}_{T} \cdot \mathbf{k}_{T}\right)} \\
& \times\left\langle P\left|\bar{\psi}(0) \gamma^{+} \psi\left(\xi^{-}, \boldsymbol{\xi}_{T}\right)\right| P\right\rangle,
\end{aligned}
$$

with the gauge link omitted. At the hadronic scale, its overlap representation reads [49]

$f_{1}^{q}\left(x, \boldsymbol{k}_{T}^{2}\right)=\frac{1}{(2 \pi)^{3}}\left[\left|\psi_{0}\left(x, \boldsymbol{k}_{T}^{2}\right)\right|^{2}+\boldsymbol{k}_{T}^{2}\left|\psi_{1}\left(x, \boldsymbol{k}_{T}^{2}\right)\right|^{2}\right]$.

We show their density plots in Fig. 9. The result for $\eta_{c}$ is on the left, while that for $\eta_{b}$ is on the right. These TMDs share the characteristics of the LF-LFWFs, i.e., they peak at the center $x=0.5$ and are narrowly distributed in $x$. Comparing the $\eta_{c}$ and $\eta_{b}$, we find the heavier bottom quarks are more centered around $x=0.5$, while more
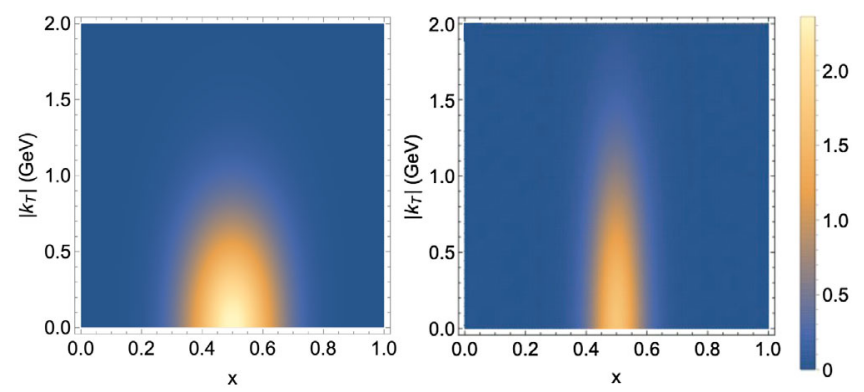

FIG. 9. Density plots of the unpolarized TMD PDF $f_{1}^{q}\left(x, \boldsymbol{k}_{T}^{2}\right)$ of $\eta_{c}$ (left) and $\eta_{b}$ (right).

broadly distributed in $\boldsymbol{k}_{T}$. Their mean transverse momentum of the valence quarks $\left\langle k_{T}\right\rangle=\int d x d^{2} \boldsymbol{k}_{T} f_{1}^{q}\left(x, \boldsymbol{k}_{T}^{2}\right)\left|\boldsymbol{k}_{T}\right|$ is $\left\langle k_{T}\right\rangle_{\eta_{c}}=0.65$ and $\left\langle k_{T}\right\rangle_{\eta_{b}}=1.02 \mathrm{GeV}$.

It is also interesting to look into the form of the transverse momentum dependence within the TMDs, which we demonstrate with Fig. 10. For the past years, the Gaussian and/or Gaussian-based $\left|\boldsymbol{k}_{T}\right|$-dependent models have been very popular in parametrizing the TMDs of pions and nucleons in the light quark sector [56,96-102]. Here we explore its validity in the heavy sector with the Gaussian form $f_{\mathrm{G}}\left(x, \boldsymbol{k}_{T}^{2}\right)=N e^{-\boldsymbol{k}_{T}^{2} /\left\langle\boldsymbol{k}_{T}^{2}(x)\right\rangle}$. We find the fitting curves (gray dotted) largely overlap with the original curves. The fitting parameters were determined to be $\left\langle\boldsymbol{k}_{T}^{2}(x=0.5)\right\rangle=(0.67 \mathrm{GeV})^{2}$ and $\left\langle\boldsymbol{k}_{T}^{2}(x=0.4)\right\rangle=$ $(0.7 \mathrm{GeV})^{2}$ for $\eta_{c}$, and $\left\langle\boldsymbol{k}_{T}^{2}(x=0.5)\right\rangle=(1.0 \mathrm{GeV})^{2}$ and $\left\langle\boldsymbol{k}_{T}^{2}(x=0.4)\right\rangle=(1.1 \mathrm{GeV})^{2}$ for $\eta_{b}$, respectively. These values are close to the total mean transverse momentum $\left\langle k_{T}\right\rangle_{\eta_{c}}=0.65$ and $\left\langle k_{T}\right\rangle_{\eta_{b}}=1.02 \mathrm{GeV}$ obtained above, suggesting weak $x$ dependence in the fitting parameter $\left\langle\boldsymbol{k}_{T}^{2}(x)\right\rangle$.

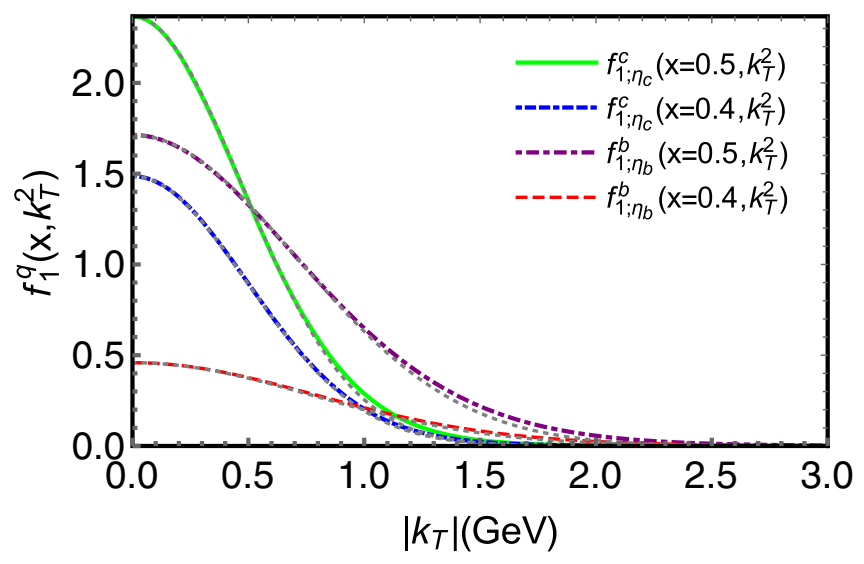

FIG. 10. The $\left|\boldsymbol{k}_{T}\right|$ dependence of $\eta_{c}$ 's and $\eta_{b}$ 's unpolarized TMD at different $x$ values. The line styles are indicated in the plot. The accompanying gray dotted curves are fitting curves in Gaussian form. 


\section{CONCLUSION}

We study the leading Fock state light-front wave functions of the light and heavy pseudoscalar mesons, using a unified framework of rainbow-ladder DS-BSEs and the light-front projection method. The LF-LFWFs of the pion at masses of 310 and $690 \mathrm{MeV}$, and those of the heavy $\eta_{c}$ and $\eta_{b}$ are reported for the first time within DS-BSEs.

The LF-LFWFs of the pion at physical mass and $310 \mathrm{MeV}$ are broadly distributed in $x$ and close to each other, while at the mass of $690 \mathrm{MeV}$, which sits at the strange quark point, i.e., the fictitious $\pi_{s \bar{s}}$, the LF-LFWFs get distinctly narrower. This trend continues in the heavy sector for the $\eta_{c}$ and $\eta_{b}$. This property is reflected in the twist-2 PDA which is the $\boldsymbol{k}_{T}$-integrated LF-LFWF. General agreement is found between our pion PDAs at different masses and those from lattice QCD [22]. In the heavy sector, where lattice QCD is absent, our PDAs of $\eta_{c}$ and $\eta_{b}$ are comparable with those from the sum rule prediction [7].

The contribution of the LF-LFWFs to meson states on the light front is further analyzed. As listed in Table I, the LF-LFWFs of pion contribute only $30 \%$ to the Fock state normalization, while in $\eta_{c}$ and $\eta_{b}$ they contribute more than $90 \%$. This indicates the existence of considerable higher Fock states in pion and, meanwhile, the dominant role of LF-LFWFs in determining heavy mesons. In particular, the latter strongly suggests the leading Fock state truncation as a reasonable means in dealing with $\eta_{c}$ and $\eta_{b}$.

We thus take the leading Fock state truncation and study the unpolarized quark GPD and TMD of $\eta_{c}$ and $\eta_{b}$ using the light-front overlap representation given in Eqs. (20) and (27). We associate the resolution scale to the sum of constituent valence (anti)quark masses, i.e., $2 m_{q}$, and study the spatial distribution of valence quarks with IPD GPD. It is found that heavier quarks are spatially more centered in heavier mesons. The study of the gravitational form factor also reveals that the heavier meson has a smaller energy radius in the light-cone frame. In the transverse momentum space, we find the heavier quark is more broadly distributed in $\boldsymbol{k}_{T}$, but more centered around longitudinal momentum fraction $x=0.5$. We also find the unpolarized TMD PDF of $\eta_{c}$ and $\eta_{b}$ can be approximated with $x$-independent Gaussian functions, as an extension to rudimentary phenomenological parametrizations of hadron TMDs in the light sector.

To conclude, this paper delivers comprehensive insights into the LF-LFWFs of pseudoscalar mesons with varying current quark mass, as well as valence quark imaging of $\eta_{c}$ and $\eta_{b}$ in both position and momentum space.

\section{ACKNOWLEDGMENTS}

We thank Jarah Evslin for helpful suggestions. This work is supported by the National Natural Science Foundation of China (under Grant No. 11905104) and the Strategic
Priority Research Program of Chinese Academy of Sciences (Grant No. XDB34030301).

\section{APPENDIX: PARAMETRIZATION OF $S(k)$ AND $\Gamma(k ; P)$}

We list the parametrization parameters of $\boldsymbol{S}(\boldsymbol{k})$ and $\boldsymbol{\Gamma}(\boldsymbol{k} ; \boldsymbol{P})$ from Eqs. (6)-(9) in Tables II and III.

TABLE II. Representation parameters for pion with different masses $m_{\pi}=130,310$, and $690 \mathrm{MeV}$. Upper part of the table: Eq. (7) - the pair $(x, y)$ represents the complex number $x+i y$. Lower part of the table: parameters of Eqs. (6), (8), and (9). (Dimensioned quantities are given in GeV.).

\begin{tabular}{lrrrrrrrrr}
\hline & \multicolumn{1}{c}{$z_{1}$} & \multicolumn{1}{c}{$m_{1}$} & \multicolumn{2}{c}{$z_{2}$} & $m_{2}$ \\
\hline$u_{\pi^{130}}$ & $(0.31,0.25)$ & $(0.52,0.27)$ & $(0.11,0.0025)$ & $(-0.81,0.71)$ \\
$u_{\pi^{310}}$ & $(0.32,0.23)$ & $(0.57,0.29)$ & $(0.11,0.016)$ & $(-0.82,0.73)$ \\
$u_{\pi^{690}}$ & $(0.31,0.26)$ & $(0.75,0.37)$ & $(0.10,1.89)$ & $(-0.89,0.011)$ \\
\hline \hline & $U_{1}$ & \multicolumn{1}{c}{$U_{2}$} & $U_{3}$ & $n_{1}$ & $n_{2}$ & $n_{3}$ & $\sigma_{1}^{i}$ & $\sigma_{2}^{i}$ & $\Lambda$ \\
\hline $\mathrm{E}_{\pi^{130}}$ & 9.88 & -5.93 & 0 & 6 & 8 & $\cdots$ & 1.76 & 0.97 & 1.7 \\
$\mathrm{~F}_{\pi^{130}}$ & 5.14 & 3.65 & 0 & 6 & 8 & $\cdots$ & 0.65 & -2.0 & 1.7 \\
$\mathrm{G}_{\pi^{130}}$ & 4.15 & -11.36 & 0 & 8 & 10 & $\cdots$ & -0.30 & -0.37 & 1.4 \\
$\mathrm{H}_{\pi^{130}}$ & 1.28 & 2.57 & 0 & 6 & 8 & $\cdots$ & 0.74 & 0.36 & 1.8 \\
$\mathrm{E}_{\pi^{310}}$ & 10.44 & -8.14 & 0 & 6 & 8 & $\cdots$ & 1.48 & 1.13 & 1.7 \\
$\mathrm{~F}_{\pi^{310}}$ & 5.01 & 2.61 & 0 & 6 & 8 & $\cdots$ & -0.085 & -2.22 & 1.7 \\
$\mathrm{G}_{\pi^{310}}$ & 2.92 & -11.38 & 0 & 8 & 10 & $\cdots$ & -0.43 & -0.55 & 1.45 \\
$\mathrm{H}_{\pi^{310}}$ & 1.04 & 2.09 & 0 & 6 & 8 & $\cdots$ & 0.20 & -0.011 & 1.86 \\
$\mathrm{E}_{\pi^{690}}$ & 11.97 & -0.99 & 0 & 6 & 8 & $\cdots$ & 1.38 & 9.20 & 2.0 \\
$\mathrm{~F}_{\pi^{690}}$ & 4.67 & 3.95 & 0 & 6 & 8 & $\cdots$ & 0.58 & -1.51 & 2.0 \\
$\mathrm{G}_{\pi^{690}}$ & 1.13 & -5.99 & 0 & 8 & 10 & $\cdots$ & -0.49 & -0.36 & 1.7 \\
$\mathrm{H}_{\pi^{690}}$ & 0.55 & -0.78 & 0 & 8 & 10 & $\cdots$ & 0.36 & 1.15 & 1.6 \\
\hline \hline
\end{tabular}

TABLE III. Representation parameters for $\eta_{c}$ and $\eta_{b}$ mesons. Upper part of table: Eq. (7) - the pair $(x, y)$ represents the complex number $x+i y$. Lower part of table: parameters of Eqs. (6), (8), and (9). (Dimensioned quantities are given in GeV.).

\begin{tabular}{ccccc}
\hline \hline & $z_{1}$ & $m_{1}$ & $z_{2}$ & $m_{2}$ \\
\hline$u$ & $(0.47,0.70)$ & $(1.84,0.54)$ & $(0.018,0.033)$ & $(-1.91,-1.16)$ \\
$b$ & $(0.47,0.66)$ & $(5.1,0.74)$ & $\ldots$ & $\ldots$ \\
\hline \hline
\end{tabular}

\begin{tabular}{lccccccccc}
\hline \hline & $U_{1}$ & $U_{2}$ & $U_{3}$ & $n_{1}$ & $n_{2}$ & $n_{3}$ & $\sigma_{1}^{i}$ & $\sigma_{2}^{i}$ & $\Lambda$ \\
\hline $\mathrm{E}_{\eta_{c}}$ & 7.14 & -8.07 & 0.18 & 5 & 6 & 1 & -1.09 & -0.91 & 2.4 \\
$\mathrm{~F}_{\eta_{c}}$ & 1.07 & 0.21 & 0.01 & 5 & 6 & 1 & -1.27 & -3.86 & 2.4 \\
$\mathrm{G}_{\eta_{c}}$ & 0.14 & -0.57 & 0.0071 & 5 & 6 & 2 & -0.87 & -0.58 & 2.0 \\
$\mathrm{H}_{\eta_{c}}$ & 0.071 & -0.079 & 0.0021 & 5 & 6 & 2 & -0.69 & 0.15 & 1.9 \\
$\mathrm{E}_{\eta_{b}}$ & 10.91 & -7.31 & 0.44 & 5 & 6 & 1 & -1.51 & -0.74 & 3.6 \\
$\mathrm{~F}_{\eta_{b}}$ & 0.55 & -0.29 & 0.013 & 5 & 6 & 1 & -1.89 & -1.40 & 3.4 \\
$\mathrm{G}_{\eta_{b}}$ & 0.0011 & -0.15 & 0.0022 & 5 & 6 & 2 & -1.75 & -1.72 & 3.3 \\
$\mathrm{H}_{\eta_{b}}$ & 0.0076 & -0.0015 & 0.00055 & 5 & 8 & 2 & -1.41 & 2.63 & 3.4 \\
\hline \hline
\end{tabular}


[1] F. Englert and R. Brout, Phys. Rev. Lett. 13, 321 (1964).

[2] P. W. Higgs, Phys. Rev. Lett. 13, 508 (1964).

[3] A. V. Radyushkin, Phys. Rev. D 56, 5524 (1997).

[4] M. Vanderhaeghen, P. A. M. Guichon, and M. Guidal, Phys. Rev. D 60, 094017 (1999).

[5] M. Beneke, G. Buchalla, M. Neubert, and C. T. Sachrajda, Nucl. Phys. B606, 245 (2001).

[6] V. V. Braguta and V. G. Kartvelishvili, Phys. Rev. D 81, 014012 (2010).

[7] T. Zhong, X.-G. Wu, and T. Huang, Eur. Phys. J. C 75, 45 (2015).

[8] A. E. Bondar and V. L. Chernyak, Phys. Lett. B 612, 215 (2005).

[9] H.-M. Choi and C.-R. Ji, Phys. Rev. D 76, 094010 (2007).

[10] V. L. Chernyak and A. R. Zhitnitsky, Phys. Rep. 112, 173 (1984).

[11] P. Ball, J. High Energy Phys. 01 (1999) 010.

[12] S. J. Brodsky, F.-G. Cao, and G. F. de Teramond, Phys. Rev. D 84, 033001 (2011).

[13] L. Chang, I. C. Clot, C. D. Roberts, S. M. Schmidt, and P. C. Tandy, Phys. Rev. Lett. 111, 141802 (2013).

[14] C. Shi, L. Chang, C. D. Roberts, S. M. Schmidt, P. C. Tandy, and H.-S. Zong, Phys. Lett. B 738, 512 (2014).

[15] C. Shi, C. Chen, L. Chang, C. D. Roberts, S. M. Schmidt, and H.-S. Zong, Phys. Rev. D 92, 014035 (2015).

[16] V. M. Braun et al., Phys. Rev. D 74, 074501 (2006).

[17] V. M. Braun, S. Collins, M. Göckeler, P. Pérez-Rubio, A. Schäfer, R. W. Schiel, and A. Sternbeck, Phys. Rev. D 92, 014504 (2015).

[18] G. S. Bali, V. M. Braun, S. Bürger, M. Göckeler, M. Gruber, F. Hutzler, P. Korcyl, A. Schäfer, A. Sternbeck, and P. Wein (RQCD), J. High Energy Phys. 08 (2019) 065; 11 (2020) 037(A).

[19] X. Ji, Y. Liu, Y.-S. Liu, J.-H. Zhang, and Y. Zhao, Rev. Mod. Phys. 93, 035005 (2021).

[20] J.-H. Zhang, J.-W. Chen, X. Ji, L. Jin, and H.-W. Lin, Phys. Rev. D 95, 094514 (2017).

[21] J.-H. Zhang, L. Jin, H.-W. Lin, A. Schäfer, P. Sun, Y.-B. Yang, R. Zhang, Y. Zhao, and J.-W. Chen (LP3 Collaboration), Nucl. Phys. B939, 429 (2019).

[22] R. Zhang, C. Honkala, H.-W. Lin, and J.-W. Chen, Phys. Rev. D 102, 094519 (2020).

[23] W. Wang, Y.-M. Wang, J. Xu, and S. Zhao, Phys. Rev. D 102, 011502 (2020).

[24] Y. Li, P. Maris, and J. P. Vary, Phys. Rev. D 96, 016022 (2017).

[25] Y. Jia and X. Xiong, Phys. Rev. D 94, 094005 (2016).

[26] W. Wang, J. Xu, D. Yang, and S. Zhao, J. High Energy Phys. 12 (2017) 012.

[27] V. V. Braguta, A. K. Likhoded, and A. V. Luchinsky, Phys. Lett. B 646, 80 (2007).

[28] M. Ding, F. Gao, L. Chang, Y.-X. Liu, and C. D. Roberts, Phys. Lett. B 753, 330 (2016).

[29] D. Binosi, L. Chang, M. Ding, F. Gao, J. Papavassiliou, and C. D. Roberts, Phys. Lett. B 790, 257 (2019).

[30] F. E. Serna, R. C. da Silveira, J. J. Cobos-Martínez, B. El-Bennich, and E. Rojas, Eur. Phys. J. C 80, 955 (2020).

[31] D. Mller, D. Robaschik, B. Geyer, F. M. Dittes, and J. Hoeji, Fortschr. Phys. 42, 101 (1994).

[32] X.-D. Ji, Phys. Rev. D 55, 7114 (1997).
[33] J. C. Collins, Acta Phys. Pol. B 34, 3103 (2003), https:// www.actaphys.uj.edu.pl/fulltext?series $=$ Reg\&vol=34\& page $=3103$.

[34] M. Burkardt, Phys. Rev. D 62, 071503 (2000); 66, 119903(E) (2002).

[35] M. Burkardt, Int. J. Mod. Phys. A 18, 173 (2003).

[36] J. C. Collins and D. E. Soper, Nucl. Phys. B194, 445 (1982).

[37] J. Collins, Foundations of Perturbative QCD (Cambridge University Press, Cambridge, England, 2013), Vol. 32.

[38] V. D. Burkert, L. Elouadrhiri, and F. X. Girod, Nature (London) 557, 396 (2018).

[39] D. E. Kharzeev, Phys. Rev. D 104, 054015 (2021).

[40] R. Wang, W. Kou, Y.-P. Xie, and X. Chen, Phys. Rev. D 103, L091501 (2021).

[41] R. Wang, W. Kou, C. Han, J. Evslin, and X. Chen, arXiv:2108.03550 [Phys. Rev. D (to be published)].

[42] A. Accardi et al., Eur. Phys. J. A 52, 268 (2016).

[43] X. Chen, F.-K. Guo, C. D. Roberts, and R. Wang, Few Body Syst. 61, 43 (2020).

[44] D. P. Anderle et al., Front. Phys. (Beijing) 16, 64701 (2021).

[45] G. P. Lepage and S. J. Brodsky, Phys. Rev. D 22, 2157 (1980).

[46] M. Diehl, Phys. Rep. 388, 41 (2003).

[47] M. Diehl, T. Feldmann, R. Jakob, and P. Kroll, Nucl. Phys. B596, 33 (2001); 605, 647(E) (2001).

[48] X.-d. Ji, J.-P. Ma, and F. Yuan, Nucl. Phys. B652, 383 (2003).

[49] B. Pasquini and P. Schweitzer, Phys. Rev. D 90, 014050 (2014).

[50] S. J. Brodsky, H.-C. Pauli, and S. S. Pinsky, Phys. Rep. 301, 299 (1998).

[51] J. Lan, K. Fu, C. Mondal, X. Zhao, and j. P. Vary, arXiv:2106.04954.

[52] G. 't Hooft, Nucl. Phys. B75, 461 (1974).

[53] H. H. Liu and D. E. Soper, Phys. Rev. D 48, 1841 (1993).

[54] T. Heinzl, Lect. Notes Phys. 572, 55 (2001).

[55] M. Burkardt, X.-d. Ji, and F. Yuan, Phys. Lett. B 545, 345 (2002).

[56] C. Shi and I. C. Clot, Phys. Rev. Lett. 122, 082301 (2019).

[57] C. Shi, K. Bednar, I. C. Cloët, and A. Freese, Phys. Rev. D 101, 074014 (2020).

[58] C. Shi, Y.-P. Xie, M. Li, X. Chen, and H.-S. Zong, arXiv:2101.09910 [Phys. Rev. D (to be published)].

[59] C. D. Roberts, arXiv:2101.08340.

[60] X. Ji and Y. Liu, arXiv:2106.05310.

[61] P. Maris and C. D. Roberts, Phys. Rev. C 56, 3369 (1997).

[62] J. C. R. Bloch, Phys. Rev. D 66, 034032 (2002).

[63] P. Maris, C. D. Roberts, and P. C. Tandy, Phys. Lett. B 420, 267 (1998).

[64] M. Blank and A. Krassnigg, Phys. Rev. D 84, 096014 (2011).

[65] T. Hilger, C. Popovici, M. Gomez-Rocha, and A. Krassnigg, Phys. Rev. D 91, 034013 (2015).

[66] C. S. Fischer, S. Kubrak, and R. Williams, Eur. Phys. J. A 51, 10 (2015).

[67] S.-x. Qin, L. Chang, Y.-x. Liu, C. D. Roberts, and D. J. Wilson, Phys. Rev. C 85, 035202 (2012). 
[68] A. Cucchieri and T. Mendes, Phys. Rev. D 78, 094503 (2008).

[69] I. L. Bogolubsky, E. M. Ilgenfritz, M. Muller-Preussker, and A. Sternbeck, Phys. Lett. B 676, 69 (2009).

[70] A. C. Aguilar, D. Binosi, and J. Papavassiliou, Phys. Rev. D 78, 025010 (2008).

[71] P. Maris and P. C. Tandy, Phys. Rev. C 60, 055214 (1999).

[72] P. Maris and P. C. Tandy, Phys. Rev. C 62, 055204 (2000).

[73] D. Jarecke, P. Maris, and P. C. Tandy, Phys. Rev. C 67, 035202 (2003).

[74] M. S. Bhagwat and P. Maris, Phys. Rev. C 77, 025203 (2008).

[75] Y.-Z. Xu, D. Binosi, Z.-F. Cui, B.-L. Li, C. D. Roberts, S.-S. Xu, and H. S. Zong, Phys. Rev. D 100, 114038 (2019).

[76] P. A. Zyla et al. (Particle Data Group Collaboration), Prog. Theor. Exp. Phys. 2020, 083C01 (2020).

[77] C. T. H. Davies, C. McNeile, E. Follana, G. P. Lepage, H. Na, and J. Shigemitsu, Phys. Rev. D 82, 114504 (2010).

[78] C. McNeile, C. T. H. Davies, E. Follana, K. Hornbostel, and G. P. Lepage, Phys. Rev. D 86, 074503 (2012).

[79] N. Souchlas, J. Phys. G 37, 115001 (2010).

[80] N. Nakanishi, Phys. Rev. 130, 1230 (1963).

[81] S. Jia and J. P. Vary, Phys. Rev. C 99, 035206 (2019).

[82] X.-d. Ji, J.-P. Ma, and F. Yuan, Eur. Phys. J. C 33, 75 (2004).

[83] C. Mezrag, H. Moutarde, and J. Rodriguez-Quintero, Few Body Syst. 57, 729 (2016).

[84] S.-S. Xu, L. Chang, C. D. Roberts, and H.-S. Zong, Phys. Rev. D 97, 094014 (2018).

[85] A. V. Efremov and A. V. Radyushkin, Theor. Math. Phys. 42, 97 (1980).
[86] C.-W. Hwang, Eur. Phys. J. C 62, 499 (2009).

[87] N. Chouika, C. Mezrag, H. Moutarde, and J. RodrguezQuintero, Few Body Syst. 58, 144 (2017).

[88] C. Shi, C. Mezrag, and H.-s. Zong, Phys. Rev. D 98, 054029 (2018).

[89] K. D. Bednar, I. C. Cloet, and P. C. Tandy, Phys. Rev. Lett. 124, 042002 (2020).

[90] J. Lan, C. Mondal, M. Li, Y. Li, S. Tang, X. Zhao, and J. P. Vary, Phys. Rev. D 102, 014020 (2020).

[91] P. Maris, AIP Conf. Proc. 892, 65 (2007).

[92] J. F. Donoghue and H. Leutwyler, Z. Phys. C 52, 343 (1991).

[93] M. V. Polyakov and P. Schweitzer, Int. J. Mod. Phys. A 33, 1830025 (2018).

[94] A. Freese and G. A. Miller, arXiv:2108.03301.

[95] A. Freese and I. C. Clot, Phys. Rev. C 100, 015201 (2019).

[96] U. D'Alesio and F. Murgia, Phys. Rev. D 70, 074009 (2004).

[97] M. Anselmino, M. Boglione, U. D’Alesio, A. Kotzinian, F. Murgia, and A. Prokudin, Phys. Rev. D 71, 074006 (2005).

[98] J. C. Collins, A. V. Efremov, K. Goeke, S. Menzel, A. Metz, and P. Schweitzer, Phys. Rev. D 73, 014021 (2006).

[99] P. Schweitzer, T. Teckentrup, and A. Metz, Phys. Rev. D 81, 094019 (2010).

[100] S. M. Aybat and T. C. Rogers, Phys. Rev. D 83, 114042 (2011).

[101] X. Wang, Z. Lu, and I. Schmidt, J. High Energy Phys. 08 (2017) 137.

[102] A. Bacchetta, F. Delcarro, C. Pisano, M. Radici, and A. Signori, J. High Energy Phys. 06 (2017) 081. 